\title{
Girino e canto de anúncio de Hypsiboas crepitans (Amphibia: Anura: Hylidae) do estado da Bahia, Brasil, e considerações taxonômicas \\ Tadpole and advertisement call of Hypsiboas crepitans (Amphibia: Anura: Hylidae) from Bahia State, Brazil, and taxonomic regards
}

Resumo: $\bigcirc$ girino e a vocalização de anúncio de Hypsiboas crepitans são descritos da Serra São José, Bahia, Brasil. $\bigcirc$ canto de anúncio é formado por três notas multipulsionadas, apresenta duração aproximada de 0,51 s e freqüência dominante de aproximadamente $0,80 \mathrm{kHz}$. Comparado com os cantos de anúncio de $H$. crepitans de outras localidades da Colômbia e do Panamá, o canto dos machos da população do estado da Bahia apresenta diferenças na freqüência dominante: mais alta que da população da Colômbia e muito mais baixa que do Panamá. Estas diferenças corroboram a hipótese de que na região amazônica ocorra outra espécie, ou mesmo, um complexo de espécies atualmente sob o nome Hypsiboas crepitans.

Palavras-chave: Hylidae. Hypsiboas crepitans. Taxonomia. Girino. Canto de anúncio.

Abstract: The tadpole and advertisement call of Hypsiboas crepitans are described from Serra São José, State of Bahia, Brazil. Three multipulsed notes composed the advertisement call; the call duration was approximately $0,51 \mathrm{~s}$ and the dominant frequency $0,80 \mathrm{kHz}$. When we compared the advertisement call of $H$. crepitans from Bahia with specimens from other localities in Colombia and Panama, we found differences in the dominant frequency: the specimens from Bahia presented higher dominant frequency than the one from Colombia and much lower than the specimens from Panama. These differences could corroborate the hypothesis that there is another species or a complex of species in the Amazonian region presently named as Hypsiboas crepitans.

Keywords: Hylidae. Hypsiboas crepitans. Taxonomy. Tadpole. Advertisement call.

\footnotetext{
Universidade Estadual de Feira de Santana. Feira de Santana, Bahia, Brasil (tituscasal@gmail.com).
}

"Iniversidade Estadual de Feira de Santana. Feira de Santana, Bahia, Brasil (florajunca@yahoo.com.br).

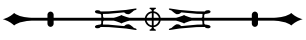




\section{INTRODUÇÃO}

A morfologia dos girinos representa uma informação relevante para o estudo da taxonomia, história natural e ecologia das espécies dos anuros (Duellman \& Trueb, 1986; Altig \& McDiarmid, 1999). Além de características morfológicas dos girinos, o canto de anúncio dos anuros, por apresentar uma série de características específicas, também é importante do ponto de vista taxonômico e tem auxiliado na determinação de espécies similares (Heyer \& Juncá, 2003; Bernal et al., 2004; Silva-Filho \& Juncá, 2006). Comparações da vocalização de anúncio inter e intraespećficas também têm sido usadas na percepção da influência da variação geográfica e no estabelecimento de padrões filogenéticos (Cocroft \& Ryan, 1995).

Hypsiboas crepitans (Wied-Neuwied, 1824) é um hilídeo cuja localidade-tipo é Tamburil, município de Condeúbas, no sul do estado da Bahia (Bokermann, 1966), mas é facilmente encontrado em todo o estado. É uma espécie de tamanho mediano (Figura 1); os machos medem entre 58,0 a 70,7 mm e fêmeas, de 60,7 a 70,0 mm (medidas a partir de espécimes de localidades do estado da Bahia). Embora seja uma espécie comum, dados na literatura sobre sua biologia e ecologia são escassos.

Esta espécie pertence ao grupo Hypsiboas faber (sensu Faivovich et al., 2005), juntamente com H. albomarginatus (Spix, 1824), H. exastis (Caramaschi \& Rodrigues, 2003), H. faber (Wied-Neuwied, 1821), H. lundii (Burmeister, 1856), H. pardalis (Spix, 1824), H. pugnax (Schmidt, 1857) e H. rosenbergi (Boulenger, 1898). Populações atribuídas a H. crepitans ocorrem no leste e nordeste do Brasil, havendo também registros em alguns outros países sul e centro americanos, como Colômbia, Venezuela, Guiana, Suriname, Guiana Francesa e Panamá (Donoso-Barros \& Ochoa, 1972; Frost, 2008). Alguns autores sugerem que as populações ocorrentes no norte da América do Sul e na América Central, identificadas também como H. crepitans, devem tratar-se de outra espécie (Donoso-Barros \& Ochoa, 1972; Kluge, 1979; Lynch \& Suárez-Mayorga, 2001; Faivovich et al., 2005).
Para contribuir com a resolução dos problemas taxonômicos, este estudo tem como objetivo descrever o girino e o canto de anúncio de $H$. crepitans a partir de dados obtidos em uma população no estado da Bahia.

\section{MATERIAL E MÉTODOS}

Os girinos de Hypsiboas crepitans foram obtidos a partir de uma desova de um casal em amplexo, encontrado junto a uma poça temporária na Serra São José (3901' W e $12^{\circ} 08^{\prime}$ S; 400 m acima do nível do mar), município de Feira de Santana, Bahia, Brasil, em 4 de junho de 2004. O casal foi levado ao laboratório e acondicionado em uma caixa plástica de $34 \mathrm{~cm}$ de comprimento, $23 \mathrm{~cm}$ de largura e $11 \mathrm{~cm}$ de altura, com água até aproximadamente cinco centímetros de altura. A fêmea desovou no mesmo dia da coleta. Os girinos foram criados e alimentados em caixas plásticas de mesmas dimensões e fixados e conservados em formalina a 10\%, em diferentes estágios de desenvolvimento.

A Serra São José encontra-se no domínio das caatingas (Ab'Saber, 1974) e é um ecótono entre floresta estacional decídua e semidecídua, floresta ombrófila densa e vegetação típica deste bioma, com presença de palmeiras. A vegetação original foi transformada em pastagens para a agricultura e pecuária, restando alguns fragmentos florestados nos cumes da serra. A paisagem é constituída por riachos intermitentes e poças temporárias, com presença também de açudes e pequenas barragens que retêm água durante todo o ano. O clima da região é seco a sub-úmido e semi-árido. Os períodos mais quentes variam entre os meses de outubro a janeiro $\left(30^{\circ} \mathrm{C}\right)$ e os mais amenos, de junho a agosto $\left(20^{\circ}\right.$ a $\left.23^{\circ} \mathrm{C}\right)$. A dinâmica das chuvas acontece com maior intensidade nos meses de novembro a maio e menor de agosto a outubro, com média pluviométrica anual de 802 mm³ (CEI-BA, 1994).

A descrição morfológica dos girinos de $H$. crepitans foi baseada em 25 indivíduos entre os estágios 35-37, de Gosner (1960), da Serra São José, Bahia. Nestes estágios, os girinos não apresentaram diferenças perceptíveis em 


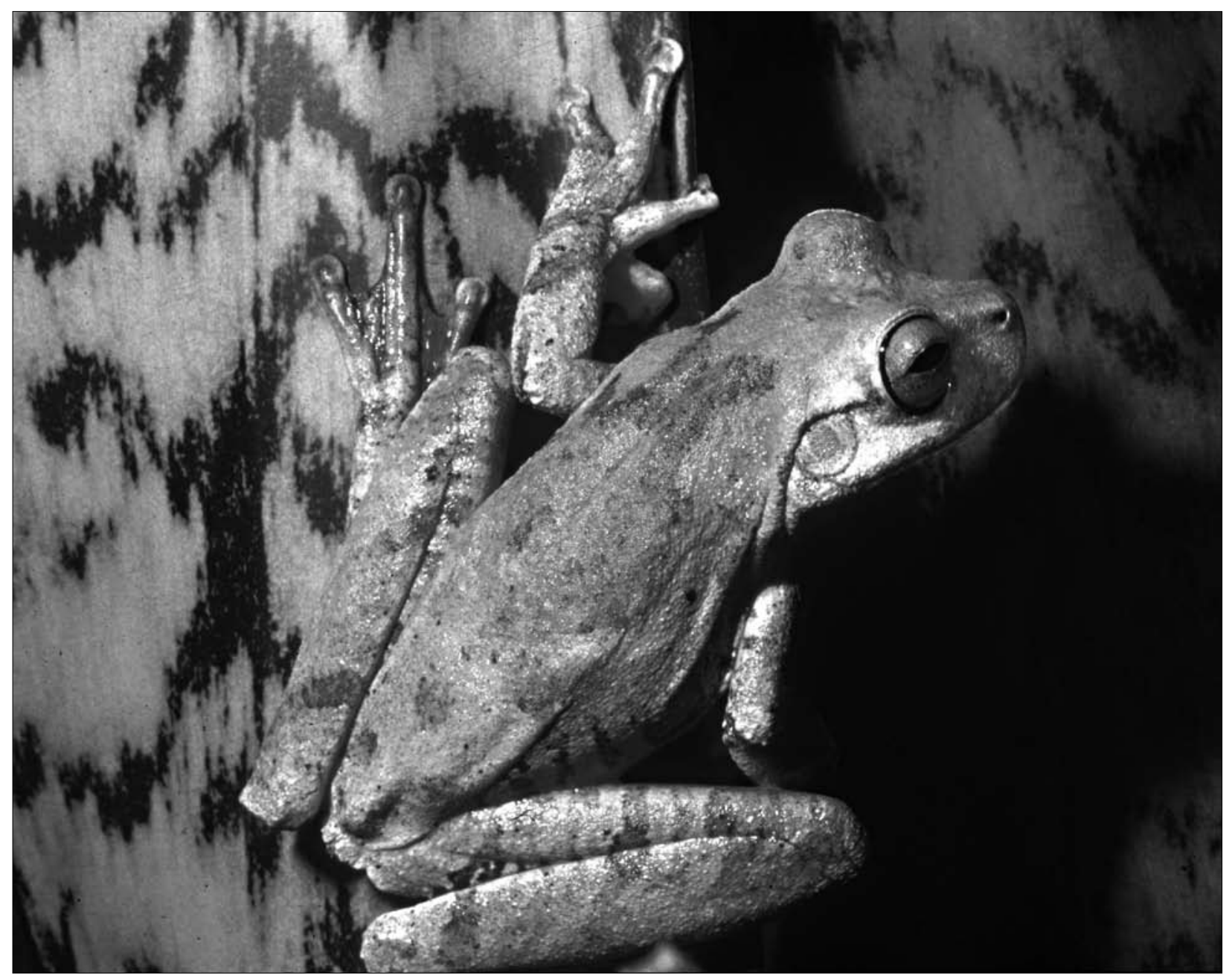

Figura 1. Exemplar de Hypsiboas crepitans oriundo da Serra São José, Feira de Sananta, Bahia. Foto: Flora Acuña Juncá.

suas proporções. Eles foram analisados e medidos em estereomicroscópio com lente micrométrica.

A fórmula dentária e o disco oral foram descritos conforme Altig \& McDiarmid (1999) e os parâmetros morfométricos seguem Wogel et al. (2000).

Foram gravados cantos de anúncio de quatro indivíduos de $H$. crepitans, oriundos da Serra São José, Bahia, utilizando um gravador digital Sony WM-D6 e microfone unidirecional Sony ECM-MS907. A temperatura do ar e da água foi de $20^{\circ} \mathrm{C}$. As características temporais (duração, intervalo, ritmo de emissão e número de pulsos por segundo) e espectral (freqüência dominante) do canto de anúncio foram analisadas através do programa Canary 1.2.4 (Charif et al., 1995), utilizando os seguintes parâmetros de resolução para o espectrograma: filter bandwidth $=174,85 \mathrm{~Hz}$; frame lenght $=23,22 \mathrm{~ms}$; time grid resolution $=1,45 \mathrm{~ms}$; frequency grid resolution $=43,0 \mathrm{~Hz}$ e FFT size $=512 \mathrm{pt}$. A terminologia dos componentes do canto seguiu Cocroft \& Ryan (1995) e Littlejohn (2001).

Os girinos analisados estão depositados no Museu de Zoologia da Universidade Estadual de Feira de Santana (MZUEFS 563). As vocalizações dos espécimes de $H$. crepitans encontram-se digitalizadas na Sonoteca

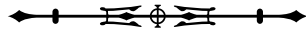


do Laboratório de Animais Peçonhentos e Herpetologia, da Universidade Estadual de Feira de Santana (SUEFS $7.4 ; 7.10 ; 7.15$ e 12.5).

\section{RESULTADOS}

A desova obtida de Hypsiboas crepitans da Serra São José apresentou, aproximadamente, 2.300 distribuídos em uma camada gelatinosa entre a superfície e o fundo d'água. Os ovos apresentaram-se pigmentados na metade exposta na superfície da água.

\section{DESCRIÇÃO DO GIRINO}

Estágios 35-37 (Gosner, 1960); Figura 2; Tabela 1 - Corpo ovóide em vistas ventral, dorsal e lateral, mais largo que alto; altura do corpo aproximadamente igual à metade do seu comprimento e largura equivalente a dois terços do comprimento do corpo. Focinho sem proeminência, arredondado em vistas ventral e dorsal; olhos grandes, dorsolaterais, com diâmetro igual a 17\% do comprimento e 25\% da largura do corpo; distância interorbital aproximadamente três vezes maior que o diâmetro do olho; narinas dorsais, pequenas, reniformes, levemente mais próximas do focinho que dos olhos; espiráculo único, curto, sinistral; tubo anal preso à nadadeira ventral e abertura lateral destra. Comprimento da cauda correspondendo a aproximadamente duas vezes o comprimento do corpo; altura da cauda ligeiramente maior que a altura do corpo; início da nadadeira dorsal no final do corpo e aproximadamente duas vezes mais alta que a nadadeira ventral; altura máxima da musculatura da cauda igual a 72\% da altura do corpo. Disco oral ânteroventral, de largura equivalente a cerca de 39\% da largura do corpo; lábio posterior desenvolvido, apresentando uma reentrância em cada canto, formando um par de bolsas, dispostas lateralmente. $\bigcirc$ disco é margeado por uma única série de fileiras de papilas, interrompida na região anterior; fórmula dentária 2(2)/4(1); quarta fileira posterior com comprimento muito menor que as demais, estando ausente em alguns exemplares. Bico córneo estreito com margens serrilhadas e processos laterais longos (Figura 2E).

Coloração em preservativo. Corpo densamente pigmentado de cor marrom acinzentada na região dorsal e lateral. Região abdominal com menos pigmentação e intestino visto por transparência. Nadadeira dorsal com alguma pigmentação preta esparsamente distribuída e nadadeira ventral transparente. Musculatura da cauda esparsamente pigmentada e exibe uma faixa preta estreita superior que se estende do início até o término da cauda. Bico córneo preto.

\section{VocalizAÇão}

Os machos de $H$. crepitans ( $n=4)$ vocalizaram em áreas abertas de vegetação típica de caatinga, em diferentes micro-hábitats, como galhos de árvores, sobre rochedos, dentro de poças temporárias e permanentes, em riachos intermitentes ou em suas margens. O número de cantos registrado para cada espécime variou de 15 a 125 cantos. Para análise, foram utilizadas amostras de 15 a 70 cantos de cada indivíduo. O número total de cantos analisados foi de 189. O canto de anúncio foi formado principalmente por três notas multipulsionadas (aproximadamente $90 \%$ dos cantos), sendo que as duas primeiras notas sempre apresentaram menor intensidade, menor número de pulsos e menor duração que a última (Figura 3). Em aproximadamente $10 \%$ dos cantos analisados, a última nota tende a fundir-se com a nota anterior, formando um canto de apenas duas notas ou, mais freqüentemente, dividir-se em sua porção inicial, formando um canto com três a cinco notas. Logo, o canto de $\mathrm{H}$. crepitans oscilou entre duas e cinco notas. $\bigcirc$ número de pulsos das primeiras notas variou de três a quatro ( $\bar{x}=3,95 \pm 0,22 ; \mathrm{n}=40$ notas), enquanto a última nota apresentou de 17 a 33 pulsos ( $\bar{x}=$ $24,15 \pm 4,31 ; n=20$ notas). O intervalo entre as notas foi de aproximadamente $0,040 \pm 0,010(n=88)$. 0 último trecho da última nota (região que nunca se separa da estrutura maior para formar outra nota) apresentou variação de dez a 26 pulsos ( $\bar{x}=18,9 \pm 3,6 ; n=111$ notas).

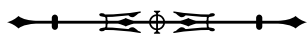


A

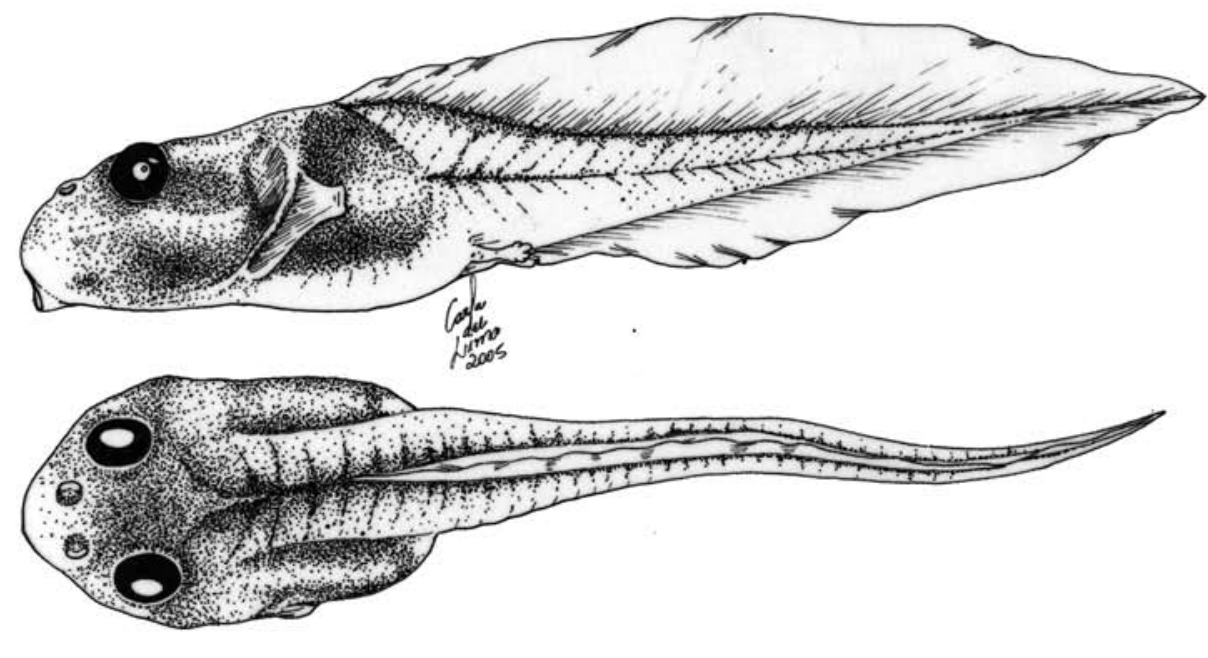

B

C

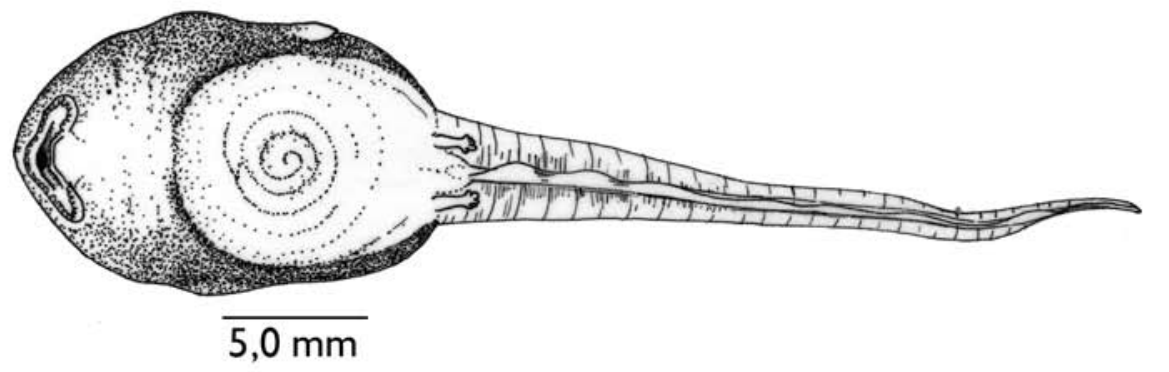

D
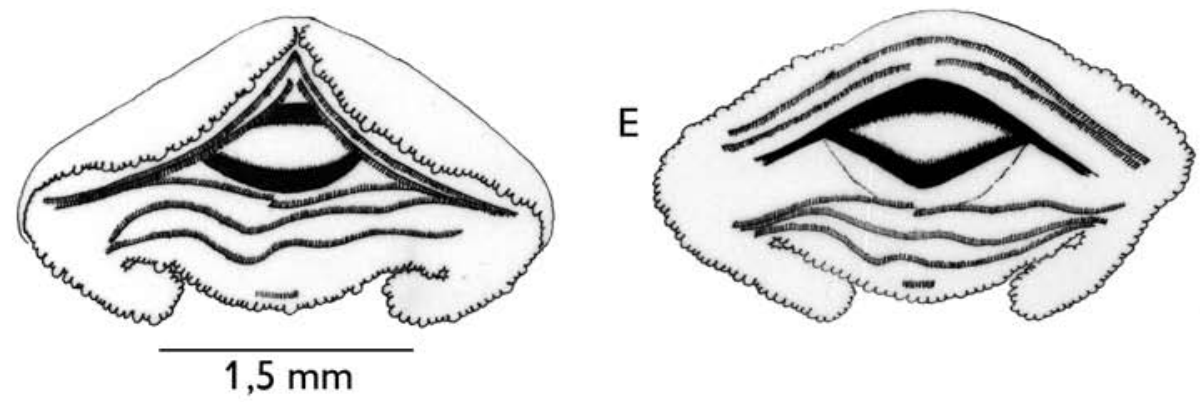

Figura 2. Girino de Hypsiboas crepitans (Feira de Santana, Bahia), em estágio de desenvolvimento 35. (A) Vista lateral; (B) Vista dorsal; (C) Vista ventral; (D) Disco oral com o lábio anterior em posição natural e (E) totalmente aberto.

Neste trecho, o ritmo de emissão dos pulsos variou de 72 a 96 pulsos por segundo $(\bar{x}=86,5 \pm 6,3 ; \mathrm{n}=111$ cantos $) .0$ canto teve uma duração média de 0,51 $\pm 0,04 s(n=189)$. O intervalo entre os cantos foi, em média, de 0,87 $\pm 0,6 \mathrm{~s}$ $(n=183)$. A taxa de emissão de cantos variou entre 24 e 52 cantos por minuto. A freqüência dominante do canto teve média de 0,80 $\pm 0,10 \mathrm{kHz}(n=189)$. Em três machos, os cantos de três notas, que representam a maioria dos cantos gravados, apresentaram-se com uma freqüência dominante $(\mathrm{kHz})$ crescente ao longo das notas (nota 1: $\bar{x}=0,66 \pm$ 0,00 ; nota $2: \bar{x}=0,70 \pm 0,00$; nota $3: \bar{x}=0,83 \pm 0,10$; $\mathrm{n}=90$ cantos). Um dos machos gravados não apresentou variação da freqüência dominante em nenhuma das notas dos cantos $(0,69 \mathrm{kHz} ; \mathrm{n}=30$ cantos $)$.

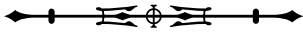


Tabela 1. Média, desvio-padrão ( \pm ) e amplitude (entre parênteses) de variação das medidas (em $\mathrm{mm}$ ) analisadas para os girinos de Hypsiboas crepitans oriundos da Serra São José, município de Feira de Santana, Bahia (estágios 35-37, n= 25).

\begin{tabular}{lr}
\hline Caracteres & \\
\hline Comprimento total & $38,8 \pm 2,6(33,1-43,4)$ \\
Comprimento do corpo & $12,7 \pm 1,1(10,3-14,6)$ \\
Altura do corpo & $6,2 \pm 0,9(4,2-7,7)$ \\
Largura do corpo & $8,3 \pm 0,9(6,6-10,2)$ \\
Comprimento da cauda & $26,1 \pm 1,8(23,8-28,9)$ \\
Altura da cauda & $7,3 \pm 0,7(5,7-9,0)$ \\
Altura da musculatura da cauda & $4,4 \pm 0,5(3,6-5,4)$ \\
Largura da musculatura da cauda & $4,3 \pm 0,4(3,6-5,1)$ \\
Altura da nadadeira dorsal & $3,1 \pm 0,3(2,6-3,5)$ \\
Altura da nadadeira ventral & $1,6 \pm 0,2(1,2-1,9)$ \\
Distância internasal & $2,9 \pm 0,2(2,4-3,3)$ \\
Distância interorbital & $6,2 \pm 0,5(5,2-7,1)$ \\
Diâmetro do olho & $2,1 \pm 0,1(1,9-2,4)$ \\
Comprimento da narina & $0,6 \pm 0,0(0,4-, 07)$ \\
Distância narina-olho & $2,8 \pm 0,2(2,3-3,1)$ \\
Distância rostro-narina & $2,5 \pm 0,2(2,0-2,8)$ \\
Largura da boca & $3,2 \pm 0,4(2,1-4,0)$ \\
\hline
\end{tabular}

\section{DISCUSSÃO}

Os girinos de Hypsiboas crepitans apresentaram-se morfologicamente muito semelhantes aos girinos de outras quatro espécies do grupo Hypsiboas faber, cujas descrições dos girinos encontram-se disponíveis: de $H$. pardalis em Bokermann (1968), de H. faber em Cei (1980), de $H$. albomarginatus em Peixoto \& Cruz (1983) e de $H$. pugnax em Chacón-Ortiz et al. (2004). Em Hypsiboas crepitans e nas quatro espécies citadas, o disco oral é margeado por uma única série de papilas, interrompida na região anterior, e o lábio posterior desenvolvido, com reentrâncias nas margens, formando um par de bolsas dispostas lateralmente. Estas cinco espécies apresentaram duas fileiras anteriores de dentículos córneos, com interrupção na primeira ou segunda fileira, e três ou quatro fileiras posteriores, sempre com interrupção na primeira fileira. Nas espécies com quatro fileiras posteriores, a quarta é muito menos extensa do que as demais e situa-se muito próxima da margem posterior do disco. Hypsiboas crepitans, $H$. albomarginatus e $H$. pugnax seguem o mesmo padrão de fórmula dentária - 2(2)/4(1), enquanto $H$. faber apresenta 2(1)/4(1) e H. pardalis, 2(1)/3(1).

Hypsiboas crepitans e $H$. pugnax são espécies muito semelhantes e costumam ser confundidas (Lynch \& SuárezMayorga, 2001; Chácon-Ortiz et al., 2004). Os girinos de ambas apresentam a cauda um pouco mais elevada que o dorso, com nadadeiras sem manchas evidentes. Já os girinos de $\mathrm{H}$. faber, $\mathrm{H}$. albomarginatus e $\mathrm{H}$. pardalis possuem manchas circulares bem características nas nadadeiras.

O canto de anúncio de H. crepitans da Serra São José apresentou o mesmo padrão de notas multipulsionadas que os cantos das populações desta espécie da região do Panamá (Kime et al., 2000) e de Ibagué, Colômbia (Bernal et al., 2004), o que faz com que o canto apresente um som áspero e inconfundível entre os ruídos noturnos da mata, como citam Donoso-Barros \& Ochoa (1972).

Neste estudo, as vocalizações de $H$. crepitans da Serra São José apresentaram grande variação na taxa de emissão de canto. No entanto, alguns autores indicam que pode haver uma grande variação intra e interespecífica quanto a este parâmetro (Gerhardt, 1994; Pough et al., 1998; Bernal et al., 2004). Por outro lado, a freqüência dominante e a taxa de emissão de pulsos são consideradas características conservativas, apresentando pouca variação entre indivíduos de mesma espécie (Littlejohn, 2001) e representando, portanto, parâmetros que auxiliam a taxonomia no nível específico (Heyer \& Juncá, 2003; Bernal et al., 2004; Silva-Filho \& Juncá, 2006). Embora resultados sobre taxa de emissão de pulso não estejam disponíveis para as populações amazônicas de H. crepitans, os espécimes da Serra São José, no estado da Bahia, apresentaram a freqüência dominante mais baixa $(0,80 \mathrm{kHz})$ que aqueles da Zona do Canal $(2,55 \mathrm{kHz}$ ) (Fouquette Jr., 1966) e do Panamá $(2,14 \mathrm{kHz})$ (Kime et al., 2000) e mais alta que os indivíduos da Colômbia (0,35 kHz) (Bernal et al., 2004). 


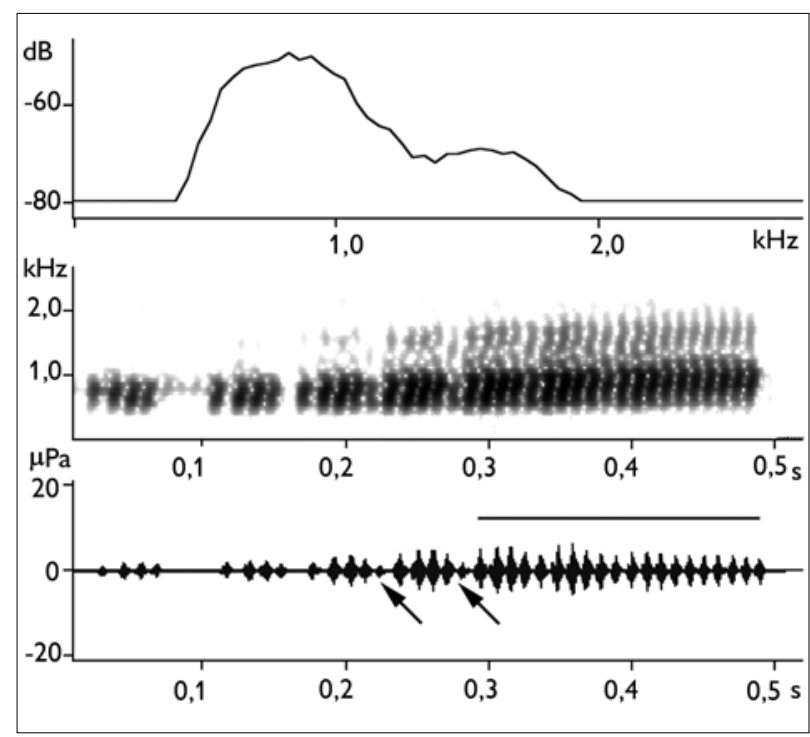

Figura 3. Canto de anúncio de Hypsiboas crepitans (SUEFS. 7.10). Temperatura do ar e água a $20^{\circ} \mathrm{C}$. Acima: Espectrograma; Meio: Sonograma; Embaixo: Oscilograma. A barra sobre o Oscilograma indica a porção da última nota que permanece constante nos cantos analisados (ver item Resultados). As setas indicam os pulsos de menor intensidade na última nota, onde em alguns cantos desaparecem, formando mais uma ou duas notas anteriores à última nota. $\mathrm{dB}=$ decibel; $\mathrm{kHz}=$ kilohertz; $\mathrm{s}=$ segundo.

Os resultados do canto de anúncio de $H$. crepitans destas diferentes populações corroboram a hipótese de que pode haver mais de uma espécie inserida neste táxon, como sugerido por outros autores (Donoso-Barros \& Ochoa, 1972; Kluge, 1979; Lynch \& Suárez-Mayorga, 2001).

\section{AGRADECIMENTOS}

Somos gratos aos colegas Flávia Maria Lima, Roseane Santiago, Márcio Borba da Silva, Alessandra Santana, Ivan Nunes e Ednei Mercês, pelo auxílio em campo. A Lucienio Mendes, por trazer o casal de H. crepitans para o Laboratório de Animais Peçonhentos e Herpetologia, da Universidade Estadual de Feira de Santana (UEFS). A Josemir de Santana e João Anfilófio, pelo apoio na manutenção dos girinos em laboratório. A Maria Conceição Lago Carneiro, pelo apoio na análise dos girinos. A Marinus Hoogmoed, pela leitura crítica do manuscrito. À UEFS, pela logística de campo. Ao Programa de Bolsas de Iniciação Científica da UEFS (PROBIC/UEFS), pela bolsa concedida ao primeiro autor.

\section{REFERÊNCIAS}

AB'SABER, A. N., 1974. O domínio morfoclimático semi-árido das caatingas brasileiras. Geomorfologia 43: 1-39.

ALTIG, R. \& R. W. MCDIARMID, 1999. Body plan. Development and morphology. In: R. ALTIG \& R. W. MCDIARMID (Eds.): Tadpoles: The Biology of Anuran Larvae: 24-51. The University of Chicago Press, Chicago and London.

BERNAL, M. H., D. P. MONTEALEGRE \& C. A. PAEZ, 2004. Estúdio de la vocalización de trece espécies de anuros del município de Ibagué, Colômbia. Revista de la Academia Colombiana de Ciencias 28(108): 385-390.

BOKERMANN, W. C., 1968. Observações sobre "Hyla pardalis" Spix (Anura, Hylidae). Revista Brasileira de Biologia 28(1): 1-5.

BOKERMANN, W. C., 1966. Lista anotada das localidades tipo de anfíbios brasileiros: 1-183. RUSP, São Paulo.

CEI, J. M., 1980. Amphibians of Argentina. Monitore Zoologico Italiano, New Series Monografia 2: 1-609.

CENTRO DE ESTATÍ́STICA E INFORMAÇÕES (CEI-BA), 1994. Informações Básicas dos Municípios Baianos: Região Paraguaçu: 1-877. CEI, Salvador.

CHACÓN-ORTIZ, A., A. D. PASCUAL \& F. GODOY, 2004. Aspectos reproductivos y desarrollo larval de Hyla pugnax (Anura: Hylidae) en el piedemonte andino de Venezuela. Revista de la Academia Colombiana de Ciencias 28(108): 391-402.

CHARIF, R. A., S. MITCHEL \& C. W. CLARK, 1995. Canary 1.2 users manual: 1-229. Cornell Laboratory of Ornithology, Ihaca.

COCROFT, R. B. \& M. J. RYAN, 1995. Patterns of advertisement call evolution in toads and chorus frogs. Animal Behaviour 49: 283-303.

DONOSO-BARROS, R. \& J. L. OCHOA, 1972. Desarrollo y evolucion larval de Hyla crepitans (Amphibia - Salientia). Boletin de la Sociedad de Biología de Concepcion 44: 117-127.

DUELLMAN, W. E. \& L. TRUEB, 1986. Biology of amphibians. Mc Graw-hill Book Company, New York.

FAIVOVICH, J. A., C. F. B. HADDAD, P. C. A. GARCIA, D. R. FROST, J. A. CAMPBELL \& W. C. WHEELER, 2005. Systematic review of the frog family Hylidae, with special reference to Hylinae: phylogenetic analysis and taxonomic revision. Bulletin of the American Museum of Natural History 294: 1-240.

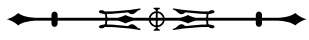


FOUQUETTE IR., M. .., 1966. Some Hylid frogs of the Canal Zone, with special reference to call structure. Caribbean Journal Science 6(3-4): 167-172.

FROST, D. R., 2008. Amphibian Species of the World. Banco de dados. Disponível em: <http://reaserch.amnh.org/herpetology/ amphibia/index.html >. Acesso em: 11 novembro 2008.

GERHARDT, H. C., 1994. The evolution of vocalization in frogs and toads. Annual Review of Ecology and Systematics 25: 293-324.

GOSNER, K. L., 1960. A simplified table for staging anuran embryos larvae with notes on identification. Herpetologica 16(2): 183-190.

HEYER, W. R. \& F. A. JUNCÁ, 2003. Leptodactylus caatingae, a new species of frog eastern Brazil (Amphibia: Anura, Leptodactylidae). Proceedings of the biological Society of Washington 116(2): 317-329.

KIME, N. M., W. R. TURNERE \& M. J. RYAN, 2000. The transmission of advertisement calls in Central American frogs. Behavioral Ecology 11(1): 71-83.

KLUGE, A. G., 1979. The gladiator frogs of Middle America and Colombia - a reevaluation of their systematics (Anura: Hylidae), Occasional papers of the museum of zoology, University of Michigan 688: 1-24.
LITTLEJOHN, N. J., 2001. Patterns of differentiation in temporal properties of acoustic signals of anurans. In: M. J. RYAN (Ed.): Anuran Communication: 102-120. Oxford University Press, New York.

LYNCH, J. D. \& A. M. SUÁREZ-MAYORGA, 2001. The distributions of the gladiator frogs (Hyla boans group) in Colombia, with comments on size variation and sympatry. Caldasia 23(2): 491-507.

PEIXOTO, O. L. \& C. A. G. CRUZ, 1983. Girinos de espécies de Hyla do grupo "albomarginata" do sudeste brasileiro (Amphibia, Anura, Hylidae). Arquivos da Universidade Federal Rural do Rio de Janeiro 6(2): 155-163.

POUGH, F. H., R. M. ANDREWS, J. E. CADLE, M. L. CRUMP, A. H. SAVITZKY \& K. D. WELLS, 1998. Herpetology. Prentice Hall, New Jersey.

SILVA-FILHO, I. S. N. \& F. A. JUNCÁ, 2006. Evidence of full species status of the neotropical leaf-frog Phyllomedusa burmeisteri bahiana (A. Lutz, 1925) (Amphibia, Anura, Hylidae). Zootaxa 1113: 51-64.

WOGEL, H., P. ABRUNHOSA \& J. P. POMBAL, 2000. Girinos de cinco espécies de anuros do sudeste do Brasil (Amphibia: Hylidae, Leptodactylidae, Microhylidae). Boletim do Museu Nacional do Rio de Janeiro 427: 1-16. 